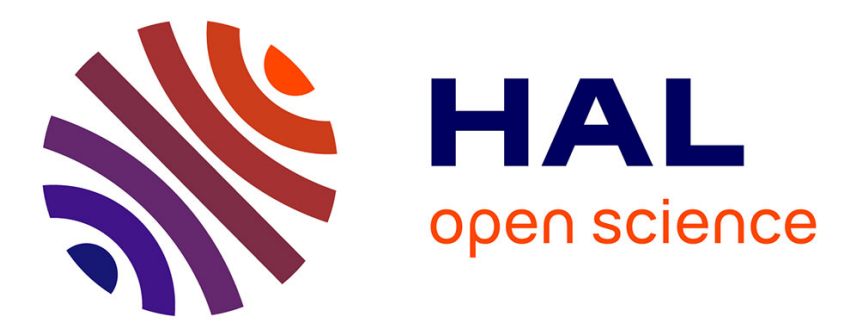

\title{
Spin-Torque-Triggered Magnetization Reversal in Magnetic Tunnel Junctions with Perpendicular Shape Anisotropy
}

N. Caçoilo, S. Lequeux, N. Strelkov, Bernard Diény, Ricardo C. Sousa, N. A. Sobolev, Olivier Fruchart, Ioan Lucian Prejbeanu, L. D. Buda-Prejbeanu

\section{To cite this version:}

N. Caçoilo, S. Lequeux, N. Strelkov, Bernard Diény, Ricardo C. Sousa, et al.. Spin-Torque-Triggered Magnetization Reversal in Magnetic Tunnel Junctions with Perpendicular Shape Anisotropy. Physical Review Applied, 2021, 16, pp.024020. 10.1103/PhysRevApplied.16.024020 . hal-02574632v2

\section{HAL Id: hal-02574632 \\ https://hal.science/hal-02574632v2}

Submitted on 7 Oct 2021

HAL is a multi-disciplinary open access archive for the deposit and dissemination of scientific research documents, whether they are published or not. The documents may come from teaching and research institutions in France or abroad, or from public or private research centers.
L'archive ouverte pluridisciplinaire HAL, est destinée au dépôt et à la diffusion de documents scientifiques de niveau recherche, publiés ou non, émanant des établissements d'enseignement et de recherche français ou étrangers, des laboratoires publics ou privés. 


\title{
Spin-Torque-Triggered Magnetization Reversal in Magnetic Tunnel Junctions with Perpendicular Shape Anisotropy
}

\author{
N. Caçoilo $\odot,{ }^{1,}{ }^{*}$ S. Lequeux, ${ }^{1}$ B.M.S. Teixeira $\odot,{ }^{1}$ B. Dieny $\odot,{ }^{1}$ R.C. Sousa $\odot,{ }^{1}$ N.A. Sobolev $\odot,{ }^{2}$ \\ O. Fruchart $\odot,{ }^{1}$ I.L. Prejbeanu® ${ }^{1}$ and L.D. Buda-Prejbeanu ${ }^{1}$ \\ ${ }^{1}$ Univ. Grenoble Alpes, CEA, CNRS, Grenoble-INP, SPINTEC, Grenoble 38000, France \\ ${ }^{2}$ I3N, Departamento de Fisica, Universidade de Aveiro, Aveiro 3810-193, Portugal
}

(Received 23 April 2021; revised 10 June 2021; accepted 29 June 2021; published 11 August 2021)

\begin{abstract}
Recently, the concept of perpendicular shape anisotropy spin-transfer-torque magnetic random-access memory (PSA STT MRAM) was proposed to enhance the thermal stability factor, $\Delta$, of the storage layer through its shape anisotropy, thus enabling the downsizing scalability of conventional STT MRAM beyond sub-20-nm technological nodes. However, this is expected to negatively impact the writing current and switching time, calling for the search for the best compromise. Here, we report a micromagnetic study of the STT-driven magnetization reversal of sub-20-nm PSA STT MRAM cells, with a particular emphasis on the technologically relevant case of $\Delta \simeq 80$, thus providing guidelines for their practical design. For an aspect ratio $(\eta)$ of the storage layer of $\eta \leq 1$, magnetization reversal obeys a macrospinlike mechanism, while for $\eta>1$ a noncoherent reversal is observed, which is characterized by buckling or nucleation of a transverse domain wall at the ferromagnet-insulator interface, which then propagates along the vertical axis. Decreasing the lateral size while maintaining a constant value of $\Delta$ implies a larger height, which is found to lead to an increase of the switching voltage. In all cases, the inverse of the switching time scales linearly with the applied voltage.
\end{abstract}

DOI: 10.1103/PhysRevApplied.16.024020

\section{INTRODUCTION}

The spin-transfer-torque magnetic random-access memory (STT MRAM) is one of the most promising emerging nonvolatile memory technologies [1-4]. It combines nonvolatility with a quasi-infinite write endurance, high speed, low power consumption, and scalability [5-7]. These properties have stimulated the commercial production of STT MRAM for a variety of stand-alone and embedded applications, in particular, ENOR flash replacement and last-level cache static random-access memory (SRAM) replacement [7-11]. While initial STT MRAM devices used an in-plane (IP) magnetization, it is shown that a perpendicular orientation of magnetization leads to a better trade-off between the thermal stability factor, $\Delta=\left(E_{B} / k_{B} T\right.$ ) (where $E_{B}$ is the energy barrier of the storage layer and $k_{B} T$ is the thermal activation energy, with $k_{B}$ being the Boltzmann constant and $T$ being the operating temperature), which determines the memoryretention time and the switching current. These devices, called perpendicular STT (PSTT) MRAM, use the interfacial perpendicular magnetic anisotropy (IPMA) existing at the interface between the storage layer (i.e., Fe-Co-B based) and the $\mathrm{MgO}$ tunnel barrier [1,5,12]. Nonetheless,

\footnotetext{
*nuno.cacoilo@cea.fr
}

the downsizing scalability of magnetic tunnel junctions (MTJs) below sub-20-nm diameters faces a fundamental challenge. Indeed, as the lateral size of the device shrinks, there is a decrease in $\Delta$ brought by the IPMA. This can be understood by considering that, at these small dimensions, reversal of the magnetic volume is almost coherent, and thus, $\Delta$ is roughly proportional to the device area. This decrease significantly reduces the retention time of the memory [13-15]. A proposal to counter this decrease is to double the IPMA by using two $\mathrm{Fe}-\mathrm{Co}(\mathrm{B}) / \mathrm{MgO}$ interfaces [16]. Still, it remains challenging to keep $\Delta>80$ at sub-20-nm diameters. A different approach consists in taking advantage of the shape anisotropy of the storage layer by increasing its thickness $(H)$ to values in the order of or larger than the storage-layer diameter. Thereby, the shape anisotropy no longer promotes easy-plane magnetization but rather further stabilizes magnetization along the perpendicular orientation. This concept of memory, named perpendicular shape anisotropy (PSA) STT MRAM, had been studied and experimentally developed simultaneously at SPINTEC [17-21] and at Tohoku University [22]. As the thickness of the storage layer increases, magnetization is expected to be more stable against thermal fluctuations due to the larger total anisotropy [17-20,22], thus allowing the cell diameter to be reduced below $20 \mathrm{~nm}$, while maintaining a sufficiently high thermal stability. 
Another significant advantage of the PSA STT MRAM is the resilience to the operating temperature [20]. This is because the magnetic properties of the thick storage layer (in particular, thermal variation of its magnetization) are much closer to those of the bulk material than those in conventional PSTT MRAM, the thin storage layer of which, only 1.5 to $2 \mathrm{~nm}$ thick, comes with an enhanced thermal decrease of magnetization. However, the increased aspect ratio $\left(\eta=\frac{H}{W}\right)$ of the storage layer in PSA STT MRAM may lead to a noncoherent reversal, higher switching voltages, and longer switching times. An understanding of the underlying mechanism of magnetization reversal is necessary to minimize these drawbacks. Here, micromagnetic simulations of the magnetization reversal mechanism of PSA MTJs induced by STT are realized, enabling the identification of different features of magnetization reversal. This study is performed by considering a prism with a fixed base width of $W=20 \mathrm{~nm}$ and an aspect ratio between 0.8 and 3. In a subsequent study, the base width of the prism is reduced to sub-20-nm dimensions, while maintaining a thermal stability factor, $\Delta$, of around 80 through the consideration of a larger aspect ratio. In both cases, the reversal mechanism and the dependency between the switching time and the applied voltage are analyzed.

\section{MICROMAGNETIC MODEL}

The magnetization dynamics is described by the Landau-Lifshitz-Gilbert-Slonczewski (LLGS) equation. We consider the material Fe-Co-B (Fig. 1), with a spontaneous magnetization of $M_{s}=1 \mathrm{MA} \mathrm{m}^{-1}$, an exchange stiffness of $A_{\mathrm{ex}}=15 \mathrm{pJ} \mathrm{m}^{-1}$, and a damping value of $\alpha=$ 0.01 [23]. The simulations are performed using the finitedifference micro3D solver with a cubic computational cell size, $\delta_{x} \times \delta_{y} \times \delta_{z}$, of $2 \times 2 \times 2 \mathrm{~nm}^{3}$ [24]. The effective magnetic field, $H_{\text {eff }}$, is calculated for each computational cell. The IPMA is implemented using an evanescent uniaxial contribution:

$$
K_{u}(z)=K_{0} \exp \left(-\frac{z}{\lambda_{K_{u}}}\right),
$$

where $\lambda_{K_{u}}$ defines the decay length of the IPMA throughout the thick layer. The value of the coefficient $K_{0}$ is adjusted, so that the incremental sum of the areal value of $K_{u}$ results in $K_{s}$, which is taken to be $1.4 \mathrm{~mJ} \mathrm{~m}^{-2}$ [23]:

$$
K_{s}=K_{0} \sum_{i=0}^{N-1} \delta_{z} \exp \left(-\frac{i \delta_{z}}{\lambda_{K_{u}}}\right)
$$

where $N$ is the number of computational-cell layers along the defined evanescent orientation $(z)$.

In Figs. 1(b) and 1(c), the equilibrium states, with and without the effect of the interfacial anisotropy, are compared for a square prism with a base width of $20 \mathrm{~nm}$ and a (a)

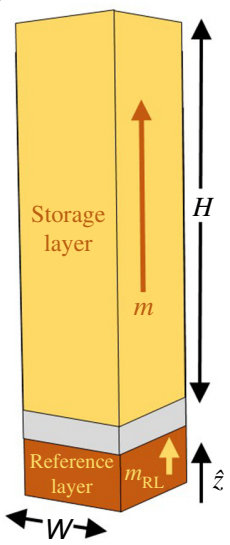

(b)

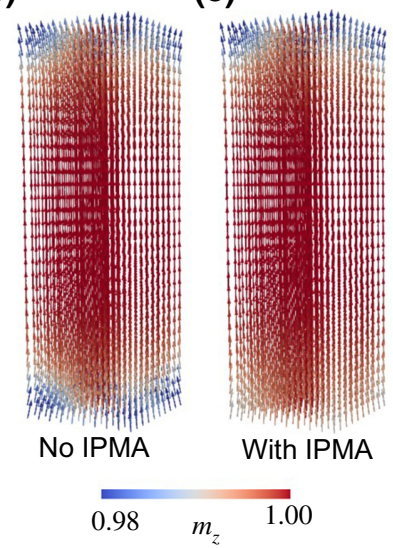

FIG. 1. (a) Three-dimensional (3D) scheme of the studied Fe-Co-B prism with height $H$ and base width $W$. Storage layer is shown with a yellowish color, tunnel barrier with a gray color, and reference layer with a reddish color. 3D equilibrium initial state of 60-nm-thick Fe-Co-B layer (b) without IPMA and (c) with IPMA. Color bar indicates normalized magnitude of magnetization along the defined $z$ direction (along the square-prism axis) in each computational cell.

height of $60 \mathrm{~nm}$. From Fig. 1(b), a flower state is observed at both the top and bottom surfaces [25]. When adding an IPMA at the bottom interface, a sturdier perpendicular orientation of the magnetization is enforced [Fig. 1(c)].

Starting from the equilibrium state with IPMA, a spinpolarized current is injected. In a thin MTJ, this effect is included in the LLGS equation as a dampinglike torque term $\left(\Gamma_{\text {STT }}^{\mathrm{IP}}\right)$ and a fieldlike torque (the latter is neglected in the following equations) [1]:

$$
\partial_{t} \mathbf{m}=-|\gamma| \mu_{0}\left(\mathbf{m} \times \mathbf{H}_{\mathrm{eff}}\right)+\alpha\left(\mathbf{m} \times \partial_{t} \mathbf{m}\right)+\Gamma_{\mathrm{STT}}^{\mathrm{IP}},
$$

where $\gamma$ is the gyromagnetic ratio, $\mu_{0}$ is the vacuum permeability, and

$$
\Gamma_{\mathrm{STT}}^{\mathrm{IP}}=-|\gamma| a_{\|} V \mathbf{m} \times\left(\mathbf{m} \times \mathbf{m}_{\mathrm{RL}}\right),
$$

where $a_{\|}$is the prefactor of the dampinglike torque, $V$ is the applied voltage, $\mathbf{m}$ is the normalized magnetization vector of the storage layer, and $\mathbf{m}_{\mathrm{RL}}$ is the normalized magnetization vector of the reference layer.

As the injection of current occurs at the bottom interface, the spin polarization is expected physically to decay exponentially through the interaction with magnetization $[26,27]$. We can model this effect, assuming that the value of $a_{\|}$decreases spatially as

$$
a_{\|}=a_{0} \exp \left(-\frac{z}{\lambda_{\mathrm{STT}}}\right),
$$

where $\lambda_{\text {STT }}$ defines the length scale of the STT decay. The value of the coefficient $a_{0}$ can be obtained by knowing 
that the averaged sum of each plane of computational cells along the material will result in a macrospin value of

$$
\left\langle a_{\|}\right\rangle=\frac{\hbar}{2|e|} \frac{\eta_{\mathrm{STT}}}{R A} \frac{1}{M_{S} H},
$$

where $\hbar$ the reduced Planck constant, $e$ is the electron charge, $\eta_{\mathrm{STT}}$ is the STT efficiency, $R A$ is the resistance-area product of the tunnel barrier, and $H$ is the total height of the storage layer. From this value, it is possible to calculate coefficient $a_{0}$ as follows:

$$
\left\langle a_{\|}\right\rangle=\frac{a_{0}}{N} \sum_{i=0}^{N-1} \exp \left(-\frac{i \delta_{\mathrm{z}}}{\lambda_{\mathrm{STT}}}\right) .
$$

Here, both decay lengths ( $\lambda_{\text {STT }}$ and $\lambda_{K_{u}}$ ) are assumed to be $1 \mathrm{~nm}$. In addition, considering the very small area of the square prism, operable PSA STT MRAM devices would require an $R A$ product in the order of $1 \Omega \mu \mathrm{m}^{2}$ to avoid excessive write voltages, which may otherwise yield dielectric breakdown $(1.2 \mathrm{~V}$ for a voltage pulse width in the order of tens of ns [23]). Since the STT efficiency is a function of the tunnel magnetoresistance (TMR) [28-30], and assuming that in our device the TMR is higher than $100 \%$, a value of $\eta_{\mathrm{STT}}=0.5$ is used.

\section{MAGNETIZATION REVERSAL DRIVEN BY STT}

We consider hereafter a set of magnetic prisms with constant base width of $20 \mathrm{~nm}$ and different pillar heights $(H=16,18,20,30,40,50$, and $60 \mathrm{~nm})$. Figure 2 shows the average magnetization along the vertical axis of the storage layer as a function of time for different heights and an applied voltage of $-3 \mathrm{~V}$. This voltage range is actually experimentally inaccessible, since it would cause dielectric breakdown of the tunnel barrier. However, one could reduce this voltage range by lowering the junction $R A$ product to values much below $1 \Omega \mu \mathrm{m}^{2}$ (as is the case in recording heads) at the expense of a lower TMR. It is observed that, for the same voltage, an increase in height results in an increase in the time it takes to start reversing the magnetic layer. For a height of $16 \mathrm{~nm}$, the switching mechanism exhibits a rather sharp variation in magnetization during reversal, whereas a slower relaxation is obtained for thicker prisms (see, e.g., $H=40$ and 50 $\mathrm{nm}$ in Fig. 2). Further increasing the height of the storage layer leads to a change in the reversal profile. Around 52 $\mathrm{nm}$, magnetization reversal starts to exhibit a shoulder; this effect is amplified as the layer height is increased to $60 \mathrm{~nm}$.

To understand the underlying mechanism of magnetization reversal of these structures, we analyze the $3 \mathrm{D}$ trajectories described by the averaged magnetization vector inside the unitary sphere, as shown in Fig. 3. Indeed, we

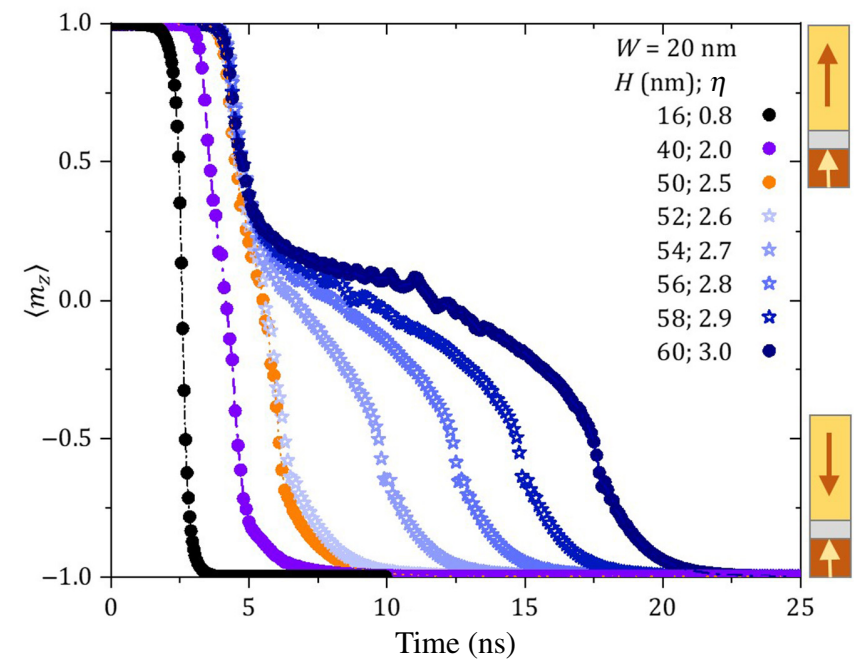

FIG. 2. Time traces of mean reduced magnetization $\left\langle m_{z}\right\rangle$ for a storage layer with a constant base width of $W=20 \mathrm{~nm}$, variable height $H$, and aspect ratio $\eta$. Applied voltage is $-3 \mathrm{~V}$.

can infer that the switching mechanism is height dependent. For a height of $16 \mathrm{~nm}$, a typical macrospin switching trajectory is observed, since the normalized magnetic moment conserves the length, and it holds for the entire trajectory curling around the unitary sphere. When increasing the height to $40 \mathrm{~nm}$, the switching trajectory no longer lies on the unitary sphere; instead, it lies inside of it, with the magnitude of the magnetic moment being smaller than one. This effect is more pronounced for a height of 60 $\mathrm{nm}$, which is also accompanied by slower magnetization dynamics. This slowing down is observed in Fig. 2 and is related to the shoulder near $\left\langle m_{z}\right\rangle \approx 0$.

We can further observe this behavior from the 3D snapshots of magnetization, shown in Fig. 4 at different time steps, for three different heights: 16,40 , and $60 \mathrm{~nm}$. Starting with the smallest height of $16 \mathrm{~nm}$, the macrospin regime is identified, since magnetization rotates coherently. Even though the model assumes the STT to decay

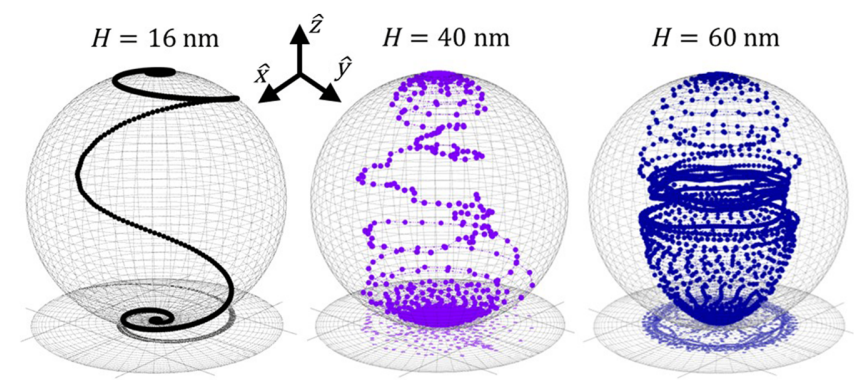

FIG. 3. Switching trajectories of magnetization of the storage layer represented on the unitary sphere. The $z$ axis represents $\left\langle m_{z}\right\rangle$ and the basal plane ( $x$ and $y$ axes) represents $\left\langle m_{x, y}\right\rangle$. Results obtained for an applied voltage of $-3 \mathrm{~V}$; pillar heights of 16, 40, and $60 \mathrm{~nm}$, and constant base width of $W=20 \mathrm{~nm}$. 


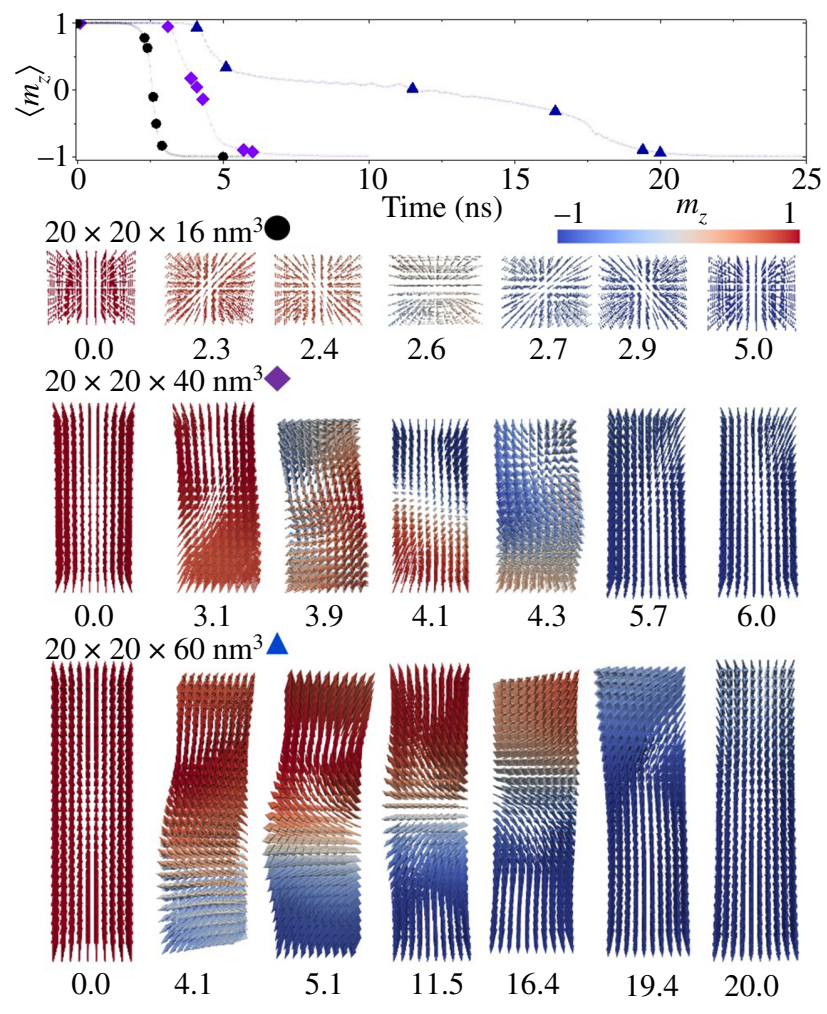

FIG. 4. Snapshots of magnetization at different time steps in ns (in bold, the time to reverse $10 \%, 50 \%$, and $90 \%$ magnetization) for 16-, 40-, and 60-nm-thick magnetic prisms with a base width of $20 \mathrm{~nm}$ and an applied voltage of $-3 \mathrm{~V}$. Color bar is linked to $m_{z}$ and time steps selected are displayed on the time trace of the average magnetization plot at the top with associated symbols.

exponentially from the $\mathrm{MgO}$ interface, at these dimensions, the exchange interactions are strong enough to insure coherent reversal. For the height of $40 \mathrm{~nm}$, magnetization reversal follows a bucklinglike mechanism, since the whole magnetic prism reacts simultaneously during reversal but in a nonuniform way [31-33]. For the largest height of $60 \mathrm{~nm}$, the nucleation of a domain wall starts at the bottom interface (where the STT is maximal). Two magnetic domains, in a tail-to-tail domain-wall configuration, are observed in the frame at $11.5 \mathrm{~ns}$. The domain wall then propagates along the vertical direction of the magnetic prism, while it rotates azimuthally in the transverse plane (as seen, for example, in Fig. 3). This mechanism of reversal is thus identified as a transverse domain-wall propagation [34].

From the curves shown in Fig. 2, it is possible to extract an important feature of the STT-driven magnetization reversal: the dependency of the switching time on the applied voltage. This switching time, $\tau_{\text {switch }}$, is defined as the time it takes to reverse half of the magnetic volume, which, in a macrospin regime, means that magnetization lies perpendicular to its initial orientation, i.e., $\left\langle m_{z}\right\rangle=0$. This is shown in Fig. 5, with an inset showing the

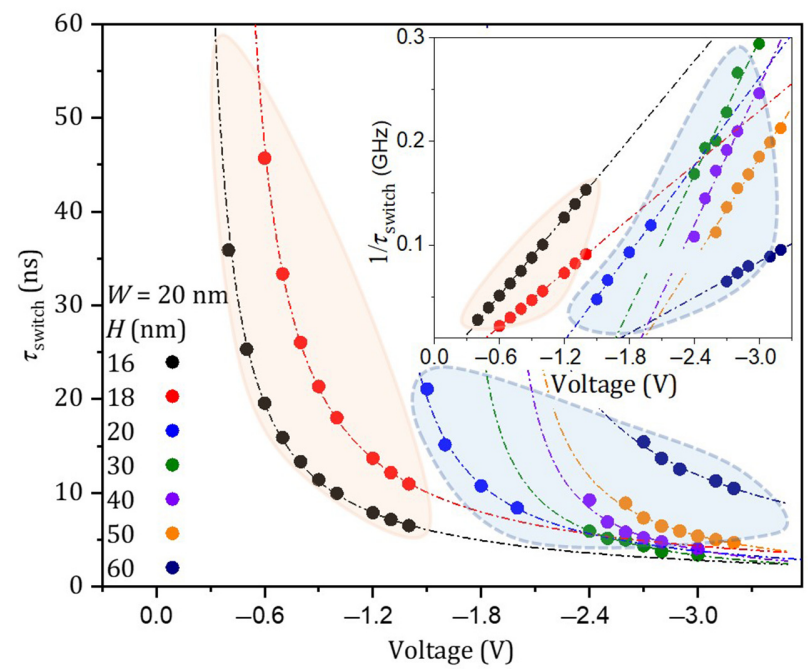

FIG. 5. Dependence of switching time on applied voltage for different heights of the storage layer and constant base width of $W=20 \mathrm{~nm}$. Coherent reversal is highlighted in light pinkish and noncoherent in light blue. Inset shows the dependency of the inverse switching time on applied voltage.

dependency of the inverse of $\tau_{\text {switch }}$ versus applied voltage. This relationship is found to be linear, and it can be ascribed to the conservation of angular momentum during the reversal process [35-37]. Moreover, the slopes of these lines are related to the magnitude of the perpendicular anisotropy field $\left(H_{\perp}\right)$ and to $\left\langle a_{\|}\right\rangle$[using Eq. (6)] [23]:

$$
\frac{1}{\tau_{\text {switch }}}=V\left(\frac{\left\langle a_{\|}\right\rangle^{2}}{H_{\perp}}\right) A-\left\langle a_{\|}\right\rangle B,
$$

where $A$ and $B$ are height-independent constants.

Indeed, fixing the base width and increasing the height leads to an increase in $H_{\perp}$, as it is dominated by the contribution of the PSA (the value of the IPMA is fixed for all heights). In addition, with increasing height, $\left\langle a_{\|}\right\rangle$ is reduced [see Eq. (6)]. As a result, the slope becomes steeper, as long as the reversal is coherent $[\eta$ of $0.8(16 \mathrm{~nm}$ height) and 0.9 (18 nm height)]. Further increasing the height of the storage layer leads to a different evolution of the slope, which reflects the noncoherent reversal behavior. This is already expected from Fig. 2, as there is a clear deviation from the macrospin regime.

Moreover, it is observed that an increase in height is accompanied by an increase in the minimum voltage required to reverse the magnetic prisms, which, as shown below, is connected with an increase in the thermal stability factor. This means that, at this width, $\eta \gg 1$ would not be feasible for a working device, because of the high switching voltage and slow reversal mechanism.

The thermal stability can be calculated using the string method [38-42], which computes the minimum-energy path that magnetization follows to reverse between its 


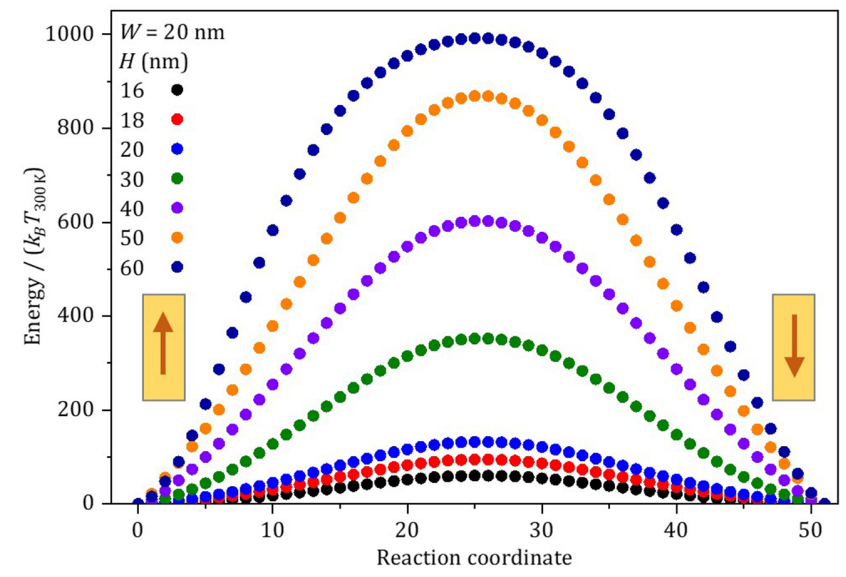

FIG. 6. Minimum-energy path between up and down states for different heights of the storage layer with constant base width of $20 \mathrm{~nm}$.

two stable states (magnetization up or down), as shown in Fig. 6. As expected, increasing the height of the storage layer significantly increases the energy barrier, leading to an increase in $\Delta$, which rapidly becomes prohibitively large for aspect ratio higher than one. Thus, it is important to take into consideration the aspect ratio of the storage layer when designing the device, as $\Delta$ is very sensitive to the layer height.

From the perspective of device applications, one should stay within a reasonable value of $\Delta$, typically in the range of 60 to 100, depending on the memory-specified retention and acceptable bit-error rate. This is the case for $\eta \lesssim 1$ at these selected material parameters. Staying within this aspect ratio also leads to a coherent reversal, avoiding the longer switching time and higher voltages needed for the high-aspect ratio pillars. As such, we study different aspect ratio combinations that give $\Delta$ of around 80 , with dimensions (both base width and height) in the sub-20$\mathrm{nm}$ design space. For these simulations, as we are using smaller heights than before, the computational cell size is reduced from 2 to $1 \mathrm{~nm}^{3}$. The selected dimensions of the storage layer comprise base widths of $18,16,14,12$, and $10 \mathrm{~nm}$, with an adjusted $\eta$ of $0.9,1.0,1.1,1.3$, and 1.8, respectively.

Despite $\Delta$ being the same, it is possible to observe two different reversal mechanisms in the 3D snapshots of magnetization, as shown in Fig. 7 at different time steps. It is found that, for $\eta \approx 1$, magnetization reversal is coherent, while, for a larger aspect ratio, noncoherent reversal occurs.

The dependency of switching time on applied voltage is shown in Fig. 8; the inset shows that $\tau_{\text {switch }}^{-1}$ again varies linearly with the applied voltage. By increasing the aspect ratio, the voltage required to reverse magnetization in the storage layer increases, even though the thermal stability factor is constant. In addition, there is no clear variation in the slope for $\eta$ of $0.9,1.0$, and 1.1, which can be attributed

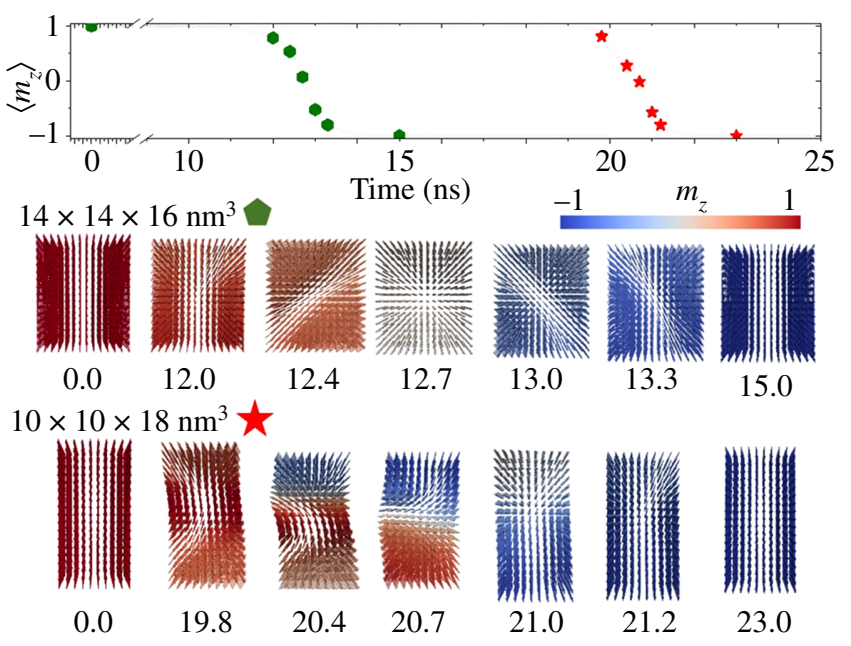

FIG. 7. Snapshots of magnetization at different time steps in ns (in bold, time to reverse $10 \%, 50 \%$, and $90 \%$ magnetization). Bottom line, pillar with $W=14 \mathrm{~nm}$ and $H=16 \mathrm{~nm}$ at an applied voltage of $-1 \mathrm{~V}$. Middle line, pillar with $W=10 \mathrm{~nm}$ and $H=$ $18 \mathrm{~nm}$ at an applied voltage of $-1.4 \mathrm{~V}$. Color bar is linked to $m_{z}$ and time steps selected are displayed on the time trace of average magnetization plot at the top with associated symbols.

to similar values of $\left\langle a_{\|}\right\rangle$and weak variation of the PSA (in comparison with the significant variation in aspect ratio seen in Fig. 5). When increasing $\eta$ from 1.1 to 1.3 and 1.8 , we can see a difference in slope, related to a nonuniform reversal. For these dimensions, the reversal is no longer strictly uniform, but rather bucklinglike (Fig. 7). This suggests that, at these typical dimensions, the type of magnetization reversal is dependent on the aspect ratio rather than on $\Delta$ itself. At first sight, it seems difficult to avoid noncoherent reversal that accompanies the high

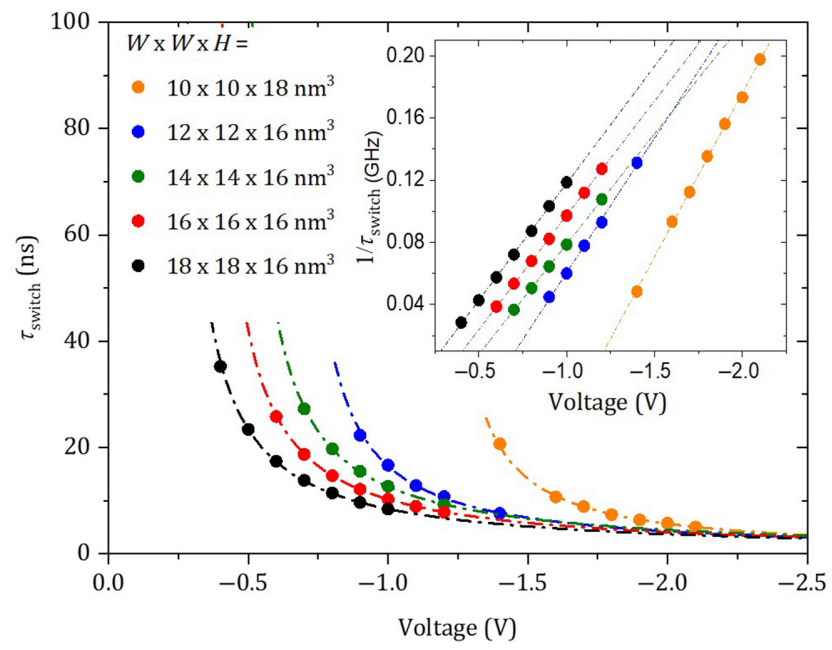

FIG. 8. Dependence of switching time on applied voltage for different studied aspect ratios $(1.8,1.3,1.1,1.0$, and 0.9). Inset shows the dependency of inverse switching time on applied voltage. 
aspect ratio required to maintain perpendicular anisotropy when going to sub-20-nm values. A possible solution to this challenge was experimentally presented by Jinnai et al. $[43,44]$, in which a thin nonmagnetic spacer layer was placed in the middle of the storage layer. With this design, it is no longer energetically favorable for noncoherent reversal to occur, leading to the possibility of having coherent reversal in the sub-20-nm PSA STT MRAM.

\section{CONCLUSIONS}

Using micromagnetic calculations, we investigate magnetization reversal in the PSA MTJ at sub-20-nm widths. For a fixed base width of $20 \mathrm{~nm}$, it is observed that, for pillars with a smaller aspect ratio, reversal exhibits a macrospinlike behavior. When increasing the height, noncoherent reversal takes place, which evolves from a bucklinglike reversal to a transverse domain-wall propagation for higher aspect ratios. The latter is associated with a slowing down of the dynamics when the domain wall is located around the middle of the storage layer. It is further observed that the inverse switching time follows a linear relationship versus applied voltage. Considering practical device applications, we then consider the specific case of a thermal stability factor of around 80 , while decreasing the width below sub-20-nm dimensions. As before, magnetization reversal evolves from macrospin to a noncoherent reversal with increasing aspect ratio. This allows us to conclude that the type of magnetization reversal mainly depends on the aspect ratio and not on $\Delta$ itself. Moreover, the linear dependency of $\tau_{\text {switch }}^{-1} \propto V$ is maintained over the whole range of memory-cell base widths investigated.

This study provides useful guidelines for the practical design of PSA STT MRAM, which can be summarized as follows: (1) the resistance times area product must be lower than that of conventional STT MRAM to keep the write voltage significantly below the barrier breakdown voltage; (2) technologically relevant $\Delta \simeq 60-100$ corresponds to an aspect ratio of about one; and (3) for $\eta \simeq 1$, magnetization reversal is nearly macrospin, with a switching time down to about 20 ns. Thus, this type of memory is not very fast but is still compatible with DRAM-type applications (for instance, battery-backed DRAM), if high memory densities can be fabricated. In addition, besides the ENOR flash replacement mentioned in Sec. I, by lowering the $R A$ product to sub- $1-\Omega \mu \mathrm{m}^{2}$ values, the switching time can be further reduced, opening prospects of applications as SRAM for low-power wearable systems, all sorts of microcontrollers, and internet-of-things devices.

\section{ACKNOWLEDGMENTS}

This work is supported by Samsung Electronics Co., Ltd. (IO190709-06540-02).
[1] D. Apalkov, B. Dieny, and J. M. Slaughter, Magnetoresistive random access memory, Proc. IEEE 104, 1796 (2016).

[2] A. V. Khvalkovskiy, D. Apalkov, S. Watts, R. Chepulskii, R. S. Beach, A. Ong, X. Tang, A. Driskill-Smith, W. H. Butler, P. B. Visscher, D. Lottis, E. Chen, V. Nikitin, and M. Krounbi, Basic principles of STT-MRAM cell operation in memory arrays, J. Phys. D: Appl. Phys. 46, 074001 (2013).

[3] Y. J. Song et al., in IEEE International Electron Devices Meeting (IEDM) (IEEE, San Francisco, CA, USA, 2018), p. 18.2.1.

[4] W. J. Gallagher, E. Chien, T. Chiang, J. Huang, M. Shih, C. Y. Wang, C. Bair, G. Lee, Y. Shih, C. Lee, R. Wang, K. Shen, J. J. Wu, W. Wang, and H. Chuang, in Symposium on VLSI Technology (IEEE, Kyoto, Japan, 2019), pp. T190.

[5] S. Ikeda, K. Miura, H. Yamamoto, K. Mizunuma, H. D. Gan, M. Endo, S. Kanai, J. Hayakawa, F. Matsukura, and H. Ohno, A perpendicular-anisotropy $\mathrm{CoFeB}-\mathrm{MgO}$ magnetic tunnel junction, Nat. Mater. 9, 721 (2010).

[6] B. Carvello, C. Ducruet, B. Rodmacq, S. Auffret, E. Gautier, G. Gaudin, and B. Dieny, Sizable room-temperature magnetoresistance in cobalt based magnetic tunnel junctions with out-of-plane anisotropy, Appl. Phys. Lett. 92, 102508 (2008).

[7] Y. J. Song, J. H. Lee, H. C. Shin, K. H. Lee, K. Suh, J. R. Kang, S. S. Pyo, H. T. Jung, S. H. Hwang, G. H. Koh, S. C. Oh, S. O. Park, J. K. Kim, J. C. Park, J. Kim, K. H. Hwang, G. T. Jeong, K. P. Lee, and E. S. Jung, in IEEE International Electron Devices Meeting (IEDM) (IEEE, San Francisco, CA, USA, 2016), p. 27.2.1.

[8] Y. K. Lee et al., in IEEE Symposium on VLSI Technology (IEEE, Honolulu, HI, USA, 2018), p. 181.

[9] Q. Dong, Z. Wang, J. Lim, Y. Zhang, M. E. Sinangil, Y. Shih, Y. Chih, J. Chang, D. Blaauw, and D. Sylvester, A 1-Mb 28-nm 1T1MTJ STT-MRAM with single-cap offset-cancelled sense amplifier and in situ self-writeTermination, IEEE J. Solid-State Circuits 54, 231 (2019).

[10] O. Golonzka et al., in IEEE International Electron Devices Meeting (IEDM) (IEEE, San Francisco, CA, USA, 2018), p. 18.1.1.

[11] J. M. Slaughter, N. D. Rizzo, J. Janesky, R. Whig, F. B. Mancoff, D. Houssameddine, J. J. Sun, S. Aggarwal, K. Nagel, S. Deshpande, S. M. Alam, T. Andre, and P. LoPresti, in International Electron Devices Meeting (IEEE, San Francisco, CA, USA, 2012), p. 29.3.1.

[12] B. Dieny and M. Chshiev, Perpendicular magnetic anisotropy at transition metal/oxide interfaces and applications, Rev. Mod. Phys. 89, 025008 (2017).

[13] C. Yoshida, T. Tanaka, T. Ataka, J. Fujisaki, K. Shimizu, T. Hirahara, H. Shitara, A. Furuya, and Y. Uehara, Size dependence of the thermal stability factor in a perpendicular $\mathrm{CoFeB} / \mathrm{MgO}$ magnetic tunnel junction studied by micromagnetic simulations, Jpn. J. Appl. Phys. 58, SBBB05 (2019).

[14] H. Sato, S. Ikeda, and H. Ohno, Magnetic tunnel junctions with perpendicular easy axis at junction diameter of less than 20 nm, Jpn. J. Appl. Phys. 56, 0802A6 (2017).

[15] L. Thomas, G. Jan, J. Zhu, H. Liu, Y.-J. Lee, S. Le, R.-Y. Tong, K. Pi, Y.-J. Wang, D. Shen, R. He, J. Haq, J. Teng, V. Lam, K. Huang, T. Zhong, T. Torng, and P.-K. Wang, Perpendicular spin transfer torque magnetic random access 
memories with high spin torque efficiency and thermal stability for embedded applications (invited), J. Appl. Phys. 115, 172615 (2014).

[16] H. Sato, M. Yamanouchi, S. Ikeda, S. Fukami, F. Matsukura, and H. Ohno, Perpendicular-anisotropy CoFeB$\mathrm{MgO}$ magnetic tunnel junctions with a $\mathrm{MgO} / \mathrm{CoFeB} / \mathrm{Ta} /$ $\mathrm{CoFeB} / \mathrm{MgO}$ recording structure, Appl. Phys. Lett. 101, 022414 (2012).

[17] N. Perrissin, S. Lequeux, N. Strelkov, A. Chavent, L. Vila, L. D. Buda-Prejbeanu, S. Auffret, R. C. Sousa, I. L. Prejbeanu, and B. Dieny, A highly thermally stable sub-20 nm magnetic random-access memory based on perpendicular shape anisotropy, Nanoscale 10, 12187 (2018).

[18] N. Perrissin, G. Gregoire, S. Lequeux, L. Tillie, N. Strelkov, S. Auffret, L. D. Buda-Prejbeanu, R. C. Sousa, L. Vila, B. Dieny, and I. L. Prejbeanu, Perpendicular shape anisotropy spin transfer torque magnetic random-access memory: Towards sub-10 nm devices, J. Phys. D: Appl. Phys. 52, 234001 (2019).

[19] N. Perrissin, N. Caçoilo, G. Gregoire, S. Lequeux, L. Tillie, N. Strelkov, A. Chavent, S. Auffret, L. D. Buda-Prejbeanu, R. C. Sousa, L. Vila, I. L. Prejbeanu, and B. Dieny, Perpendicular shape anisotropy spin transfer torque-MRAM: Determination of pillar tilt angle from 3D stoner-wohlfarth astroid analysis, J. Phys. D: Appl. Phys. 52, 505005 (2019).

[20] S. Lequeux, N. Perrissin, G. Grégoire, L. Tillie, A. Chavent, N. Strelkov, L. Vila, L. D. Buda-Prejbeanu, S. Auffret, R. C. Sousa, I. L. Prejbeanu, E. D. Russo, E. Gautier, A. P. Conlan, D. Cooper, and B. Dieny, Thermal robustness of magnetic tunnel junctions with perpendicular shape anisotropy, Nanoscale 12, 6378 (2020).

[21] S. Lequeux, T. Almeira, N. Caçoilo, A. Palomino, L. Prejbeanu, R. Sousa, D. Cooper, and B. Dieny, in IEEE International Memory Workshop (IMW) (IEEE, Dresden, Germany, 2021).

[22] K. Watanabe, B. Jinnai, S. Fukami, H. Sato, and H. Ohno, Shape anisotropy revisited in single-digit nanometer magnetic tunnel junctions, Nat. Commun. 9, 663 (2018).

[23] B. Dieny and I. L. Prejbeanu, in Introduction to Magnetic Random-Access Memory (John Wiley \& Sons, Ltd, Hoboken, New Jersey, USA, 2017), Chap. 5, p. 101.

[24] L. D. Buda, I. L. Prejbeanu, U. Ebels, and K. Ounadjela, Micromagnetic simulations of magnetisation in circular cobalt dots, Comput. Mater. Sci. 24, 181 (2002).

[25] M. E. Schabes and H. N. Bertram, Magnetization processes in ferromagnetic cubes, J. Appl. Phys. 64, 1347 (1988).

[26] M. Chshiev, A. Manchon, A. Kalitsov, N. Ryzhanova, A. Vedyayev, N. Strelkov, W. H. Butler, and B. Dieny, Analytical description of ballistic spin currents and torques in magnetic tunnel junctions, Phys. Rev. B 92, 104422 (2015).

[27] J. Grollier, A. Chanthbouala, R. Matsumoto, A. Anane, V. Cros, F. Nguyen van Dau, and A. Fert, Magnetic domain wall motion by spin transfer, C. R. Phys. 12, 309 (2011).

[28] J. Z. Sun et al., Spin-torque switching efficiency in CoFeB$\mathrm{MgO}$ based tunnel junctions, Phys. Rev. B 88, 104426 (2013).
[29] J. C. Slonczewski and J. Z. Sun, Theory of voltagedriven current and torque in magnetic tunnel junctions, J. Magn. Magn. Mater. Proc. 17th Int. Conf. Magn. 310, 169 (2007).

[30] J. Z. Sun and D. C. Ralph, Magnetoresistance and spintransfer torque in magnetic tunnel junctions, J. Magn. Magn. Mater. 320, 1227 (2008).

[31] A. Aharoni, Magnetization buckling in elongated particles of coated iron oxides, J. Appl. Phys. 63, 4605 (1988).

[32] A. Aharoni and S. Shtrikman, Magnetization curve of the infinite cylinder, Phys. Rev. 109, 1522 (1958).

[33] R. Hertel and J. Kirschner, Magnetization reversal dynamics in nickel nanowires, Phys. B: Condens. Matter Proc. Fourth Intional Conf. Hysteresis Micromagnetic Model. 343, 206 (2004).

[34] A. Thiaville, Y. Nakatani, J. Miltat, and Y. Suzuki, Micromagnetic understanding of current-driven domain wall motion in patterned nanowires, EPL 69, 990 (2005).

[35] K. Garello, C. O. Avci, I. M. Miron, M. Baumgartner, A. Ghosh, S. Auffret, O. Boulle, G. Gaudin, and P. Gambardella, Ultrafast magnetization switching by spin-orbit torques, Appl. Phys. Lett. 105, 212402 (2014).

[36] H. Liu, D. Bedau, J. Z. Sun, S. Mangin, E. E. Fullerton, J. A. Katine, and A. D. Kent, Dynamics of spin torque switching in all-perpendicular spin valve nanopillars, J. Magn. Magn. Mater. 358-359, 233 (2014).

[37] D. C. Worledge, G. Hu, D. W. Abraham, J. Z. Sun, P. L. Trouilloud, J. Nowak, S. Brown, M. C. Gaidis, E. J. O'Sullivan, and R. P. Robertazzi, Spin torque switching of perpendicular $\mathrm{Ta} / \mathrm{CoFeB} / \mathrm{MgO}$-based magnetic tunnel junctions, Appl. Phys. Lett. 98, 022501 (2011).

[38] H. Forster, N. Bertram, X. Wang, R. Dittrich, and T. Schrefl, Energy barrier and effective thermal reversal volume in columnar grains, J. Magn. Magn. Mater. 267, 69 (2003).

[39] E. Weinan, W. Ren, and E. Vanden-Eijnden, Energy landscape and thermally activated switching of submicron-sized ferromagnetic elements, J. Appl. Phys. 93, 2275 (2003).

[40] G. D. Chaves-O'Flynn, A. D. Kent, and D. L. Stein, Micromagnetic study of magnetization reversal in ferromagnetic nanorings, Phys. Rev. B 79, 184421 (2009).

[41] G. D. Chaves-O'Flynn, D. Bedau, E. Vanden-Eijnden, A. D. Kent, and D. L. Stein, Stability of $2 \pi$ domain walls in ferromagnetic nanorings, IEEE. Trans. Magn. 46, 2272 (2010).

[42] M. F. Carilli, K. T. Delaney, and G. H. Fredrickson, Truncation-based energy weighting string method for efficiently resolving small energy barriers, J. Chem. Phys. 143, 054105 (2015).

[43] B. Jinnai, J. Igarashi, K. Watanabe, E. C. I. Enobio, S. Fukami, and H. Ohno, in IEEE International Electron Devices Meeting (IEDM) (IEEE, San Francisco, CA, USA, 2020).

[44] B. Jinnai, J. Igarashi, K. Watanabe, E. C. I. Enobio, S. Fukami, and H. Ohno, Coherent magnetization reversal of a cylindrical nanomagnet in shape-anisotropy magnetic tunnel junctions, Appl. Phys. Lett. 118, 082404 (2021). 